\title{
Mental health-related impact applying a scalp cooling technology solution in chemotherapy: a scoping review
}

\author{
Jessica Ranieri \\ University of L'Aquia \\ Federica Guerra \\ University of L'Aquila \\ Dina Di Giacomo ( $\nabla$ dina.digiacomo@cc.univaq.it ) \\ University of L'Aquila https://orcid.org/0000-0001-8189-2052
}

\section{Research Article}

Keywords: mental health, scalp cooling system, chemotherapy, clinical psychology, psychoncology

Posted Date: March 11th, 2021

DOl: https://doi.org/10.21203/rs.3.rs-223562/v1

License: (a) This work is licensed under a Creative Commons Attribution 4.0 International License. Read Full License 


\section{Abstract}

Background and Objective

A scalp cooling system, particularly, a digitised scalp-cooling system, is currently assuming an important role during adjuvant treatment within the oncological setting, promising the prevention of hair loss and turning an efficient procedure to reinforce the mental health of patients during chemotherapy by preventing chemotherapy-induced alopecia (CIA). We conducted a scoping review to map the literature on the efficacy of the scalp cooling technology in chemotherapy. We focused on the efficacy of the scalp cooling system in preventing $\mathrm{CIA}$ and the associated mental health effects, such as reducing mood changes, depression, anxiety, anger, sleep disorders, and fatigue.

Methods

We performed a systematic search of MEDLINE via PubMed, Embase, Web of Science, PsycINFO, and Scopus in May 2020. We conducted a descriptive analysis of the characteristics of the included literature. Based on the exclusion/inclusion criteria, a total of 17 articles were included in the scoping review process.

Results

This review demonstrated the lack of research on the efficacy of adopting the scalp cooling system as preventive care for the well-being of patients through the prevention of their hair loss. Nevertheless, research on this topic is progressively increasing, and future studies should address specific key points.

Conclusion

This study aimed to identify emerging and urgent research domains in order to design better clinical outcomes for innovative solutions, integrating the physical and mental health of oncological patients in survivorship.

\section{Introduction}

Cancer patients face a number of disease-related challenges that lead to clinically relevant mood disorders, psychological distress, and depression (Linden et al., 2012; Seiler \& Jenewein, 2019; Di Giacomo et al., 2019). In primary treatment, patients have to deal with chemotherapy and its associated burdensome physical effects, as well as effects on body image (Mehnert et al., 2018). One such side-effect is chemotherapy-induced alopecia, a temporary form of hair loss that could influence the mental health of patients who suffer from it. In order to support patients dealing with the side effects of adjuvant treatments, the scalp hypothermia technique was introduced as a preventative measure against the development of CIA. Technological innovations in clinical medicine, specifically in oncological treatments, allowed for the introduction of a scalp cooling system aimed at preventing hair loss during chemotherapy treatment. Currently, DigniCap, a digitized scalp-cooling system, is assuming an important role in the clinical setting during adjuvant treatment, promising hair loss prevention and allowing an efficient procedure to reinforce the mental health of patients during chemotherapy by avoiding CIA. DigniCap comprise a refrigerator unit and a control unit integrated into a mobile cabinet and connected to a tight-fitting cooling cap. This system maintains a constant scalp temperature of $+5^{\circ} \mathrm{C}$ during all chemotherapy treatments (lasting during the entire session, for minimum of 4 hours or longer, as part of the planned chemotherapy regimen). The application of this digital solution in medical treatments is primarily based on experimentation data demonstrating reduction in the rate of hair loss as the primary outcome for a higher quality of life during chemotherapy and post-primary treatment (Giarratano et al., 2019). The increasing application of scalp cooling systems in chemotherapy has led to the emergence of an innovative solution in clinical medicine related to mental health during oncological treatments.

The aim of this study is to map the literature regarding the mental health influence of the DigniCap system's application in chemotherapy treatments, defining the strengths and weaknesses of the research topic. This scoping review focuses on the efficacy of the DigniCap system in preventing CIA as well as its influence on mental health, particularly, in reducing mood changes, depression, anxiety, anger, sleep disorders, and fatigue. Specifically, we wanted to quantify and synthetize the studies 
conducted thus far regarding the efficacy of the mental health effect of the digitised scalp cooling systems and highlight the emerging key points for future research protocols. Ideally, our findings could aid in the harmonisation of the implementation of cancer care toward high quality of life in survivorship. The scope of this review is to maximise the value of previously published findings and to harmonise future study approaches, drawing on potential future challenges and prompting key components for future research.

\section{Methods}

We conducted a scoping review to map the literature on the efficacy of the DigniCap technology in chemotherapy. The framework outlined by Arksey and O'Malley (2005) in their methodological paper on scoping studies was adopted. Our aim was 1) to summarise and disseminate current available evidence for the impact of DigniCap on the psychological needs of oncological patients, and 2) to propose key points for future research.

Specifically, the research objectives of this review have been:

1. to summarise the current evidence on the DigniCap's impact on the quality of life and wellness of patients;

2. to identify gaps in the literature that may require further research; and

3. to identify relevant research questions, particularly, the determinants of future implementation approaches.

\section{Search strategy}

We performed a systematic search of MEDLINE via PubMed, Embase, Web of Science, PsycINFO, and Scopus in May 2020 with the terms 'dignicap', 'scalp cooling', 'scalp hypothermia', and 'hypothermic cap', combined with 'quality of life', 'wellness', and 'clinical psychology', published from May 2000 to May 2020.

\section{Inclusion and exclusion criteria}

We included all literature related to the Dignicap system published in English until 30 May 2020 without restrictions. This comprised reviews, clinical studies, basic research, epidemiological studies, and clinical trials. However, we excluded news reports that were not published in scientific journals and articles whose full text we were unable to access despite contacting the authors.

\section{Article selection and data extraction}

Two reviewers independently screened all titles, abstracts, and full texts and resolved disagreements by consensus or consultation with a third reviewer. The following information were extracted: (i) title, (ii) authors, (iii) journal, (iv) publication or posted date, (v) type of article/study, (vi) topic, (viii) objectives of study, and (ix) results.

\section{Statistical analysis}

We conducted a descriptive analysis of the characteristics of the included literature. We described the source, publication date, type of article/study, and topic of article/study to examine the existing gaps in research. We categorised the literature into reviews, clinical studies (including randomised controlled trials and observational studies), basic research, epidemiological studies, editorial comments, and other categories if identified. We conducted this scoping review in accordance with the PRISMA-ScR Checklist (Tricco et al., 2018).

\section{Results}

\section{Search results}

Our initial search returned n 35 papers from our database. After duplicate and inadequate articles were removed, we identified $\mathrm{n}$ 20 records that underwent title and abstract review for relevance. Then, three articles were excluded during full-text review.

Figure 1 illustrates the study selection flowchart.

\section{Characteristics of included articles/studies}


The articles included in the scoping review were cohort studies, review studies, clinical trials, and basic research.

\section{Source of articles/studies}

All articles/studies have been published in peer-reviewed journals. The journal with the highest number of published articles was Cancer Research $(n=4,23.5 \%)$. 
Table 1

a - Publishers of articles/studies

\begin{tabular}{|c|c|c|c|}
\hline \multicolumn{2}{|l|}{ Characteristics of articles/studies } & \multirow{2}{*}{$\begin{array}{l}\text { Number } \\
4\end{array}$} & \multirow{2}{*}{$\begin{array}{l}\% \\
23,5\end{array}$} \\
\hline Platform Publication: Journal $(=17)$ & Cancer Research & & \\
\hline & Breast Cancer Research and Treatment & 2 & 11,8 \\
\hline & JAMA & 1 & 5,9 \\
\hline & The Breast Journal & 1 & 5,9 \\
\hline & Breast Journal & 1 & 5,9 \\
\hline & Supportive Care in Cancer & 1 & 5,9 \\
\hline & Springerplus & 1 & 5,9 \\
\hline & Asia-Pacific Journal of Oncology Nursing & 1 & 5,9 \\
\hline & Expert Review of Dermatology & 1 & 5,9 \\
\hline & British Journal of Cancer & 1 & 5,9 \\
\hline & Reviews on Recent Clinical Trials & 1 & 5,9 \\
\hline & Future Oncology & 1 & 5,9 \\
\hline & Current Oncology & 1 & 5,9 \\
\hline \multirow[t]{7}{*}{ First Author' Country } & Italy & 5 & 29,4 \\
\hline & USA & 4 & 23,5 \\
\hline & Germany & 2 & 11,8 \\
\hline & Australia & 2 & 11,8 \\
\hline & The Netherlands & 2 & 11,8 \\
\hline & Mexico & 1 & 5,9 \\
\hline & Switzerland & 1 & 5,9 \\
\hline \multirow[t]{13}{*}{ Publication or posted date } & November-2019 & 1 & 5,9 \\
\hline & October-2019 & 1 & 5,9 \\
\hline & July-2019 & 1 & 5,9 \\
\hline & February-2019 & 1 & 5,9 \\
\hline & January-2019 & 1 & 5,9 \\
\hline & September-2018 & 1 & 5,9 \\
\hline & July-2018 & 1 & 5,9 \\
\hline & February-2018 & 1 & 5,9 \\
\hline & February-2017 & 2 & 11,8 \\
\hline & December-2016 & 1 & 5,9 \\
\hline & April-2016 & 1 & 5,9 \\
\hline & February-2016 & 1 & 5,9 \\
\hline & March-2015 & 1 & 5,9 \\
\hline
\end{tabular}




\begin{tabular}{|llll|}
\hline Characteristics of articles/studies & \multicolumn{2}{c|}{ Number } & $\%$ \\
\hline & September-2014 & 1 & 5,9 \\
\cline { 2 - 4 } & December-2012 & 1 & 5,9 \\
\cline { 2 - 4 } & February-2011 & 1 & 5,9 \\
\hline
\end{tabular}

\section{Type of articles/studies}

The types of published articles are reported in Table $1 \mathrm{~b}$.

The platform of publication is generally on oncological outlook (Current Oncology, Cancer Research, and Breast Cancer Research \& Treatment), involving mostly Italian and American authors. Moreover, looking at the posted date of publication, interest in this topic is seen to be increasing progressively over time.

Table 1

b - Characteristics of DigniCap articles/studies in breast cancer chemotherapy.

\begin{tabular}{|c|c|c|c|}
\hline \multicolumn{2}{|c|}{ Characteristics of articles/studies } & \multirow{2}{*}{$\begin{array}{l}\text { Number } \\
14\end{array}$} & \multirow{2}{*}{$\begin{array}{l}\% \\
82,4\end{array}$} \\
\hline Topic & Efficacy on CIA Prevention & & \\
\hline & Quality of Life & 9 & 52,9 \\
\hline & Emotional features (anxiety, depression, body image, distress, satisfaction) & 7 & 41,2 \\
\hline & Tolerability and safety & 4 & 23,5 \\
\hline & Attitude \& knowledge on SC & 3 & 17,6 \\
\hline \multirow[t]{4}{*}{ Type of articles/studies } & Cohort study & 6 & 46,2 \\
\hline & Basic research & 3 & 23,1 \\
\hline & Clinical trial & 2 & 15,4 \\
\hline & Review & 2 & 15,4 \\
\hline \multirow[t]{3}{*}{ Type of study poster } & Cohort study & 2 & 50 \\
\hline & Clinical trial & 1 & 25 \\
\hline & Basic Research & 1 & 25 \\
\hline
\end{tabular}

Table $1 \mathrm{~b}$ reports the typologies of the studies conducted to date. The cohort study design seemed to be more widely applied than the other study designs. By contrast, there was a lower rate of published basic research and clinical trial study designs. Finally, the research topic that had been more relevant in the literature was the investigation of CIA prevention efficacy. Following this was the focus on the impact on psychological needs (quality of life and emotional traits).

In Table 2, screened articles highlighting the details of each research protocol were grouped into three study designs: clinical trials $(n=3)$, cohort studies $(n=8)$, and basic research $(n=4)$. Clinical trials (Cigler et al., 2017; Smetanay et al., 2018; Munzone et al., 2019) have been conducted mostly from a multicentric perspective and observational studies.

Table 2 - Details of experimental studies anazlized in this scoping review 


\begin{tabular}{|c|c|c|c|c|c|c|c|c|c|}
\hline $\begin{array}{l}\text { TYPE OF } \\
\text { STUDY }\end{array}$ & AUTHORS & KEYWORD AIM & TOPIC & $\begin{array}{l}\text { STUDY } \\
\text { DESIGN }\end{array}$ & $\begin{array}{c}\text { STATISTICAL } \\
\text { ANALYSIS }\end{array}$ & SAMPLE SIZE & $\begin{array}{l}\text { TARGET } \\
\text { SAMPIE }\end{array}$ & TESTS & OUTCOMES \\
\hline \multirow[t]{3}{*}{$\begin{array}{l}\text { CLINICAL } \\
\text { TRIAL }\end{array}$} & $\begin{array}{l}\text { Cigler et al. } \\
(2017)\end{array}$ & $\begin{array}{l}\text { HAIR LOSS + } \\
\text { QoL }\end{array}$ & CIA + QoL & $\begin{array}{l}\text { Prospective } \\
\text { non } \\
\text { randomized } \\
\text { open label; } \\
\text { treatment } \\
\text { control } \\
\text { multicenter } \\
\text { study }\end{array}$ & $\begin{array}{l}\text { Risk ratios } \\
\text { using } \\
\text { Mantel- } \\
\text { Haenszel } \\
\text { random } \\
\text { effects model } \\
\text { use to assess } \\
\text { differences }\end{array}$ & $\begin{array}{l}\text { N.117 } \\
\text { patients } \\
\text { (N.101 } \\
\text { received } \\
\text { scalp cooling } \\
\text { using } \\
\text { Dignicap and } \\
\text { N.16 were } \\
\text { controls (no } \\
\text { scalp cooling) }\end{array}$ & $\begin{array}{l}\text { Women, age } \\
>18 \text { years, } \\
\text { stage I or II } \\
\text { breast cancer } \\
\text { receiving } \\
\text { neo/adjuvant } \\
\text { chemotherapy } \\
\text { regimens. } \\
\text { Women who } \\
\text { chose not to } \\
\text { undergo scalp } \\
\text { cooling were } \\
\text { enrolled as } \\
\text { controls. }\end{array}$ & $\begin{array}{l}\text { BR23; BIS; hair } \\
\text { loss self- } \\
\text { assessment } \\
\text { with treatment } \\
\text { success } \\
\text { defined as } \\
<=50 \text { hair loss }\end{array}$ & $\begin{array}{l}66.3 \% \text { of } \\
\text { patients using } \\
\text { Dignicap } \\
\text { achieved } \\
\text { treatment } \\
\text { success. } \\
\text { Women with } \\
\text { BC receiving } \\
\text { DIGNICAP vs } \\
\text { control during } \\
\text { chemotherapy: } \\
\text { were } \\
\text { significantly } \\
\text { less likely to } \\
\text { lose >50\% of } \\
\text { their hair; felt } \\
\text { significantly } \\
\text { more } \\
\text { physically } \\
\text { attractive; } \\
\text { were } \\
\text { significantly } \\
\text { less } \\
\text { dissatisfied } \\
\text { with their } \\
\text { appearance } \\
\text { when dressed; } \\
\text { regarded the } \\
\text { importance of } \\
\text { hair } \\
\text { significantly } \\
\text { more } \\
\text { iinaing }\end{array}$ \\
\hline & $\begin{array}{l}\text { Smetan et } \\
\text { al. } \\
(2018)\end{array}$ & $\begin{array}{l}\text { EFFICACY + } \\
\text { TOLERABILITY } \\
+ \text { QoL }\end{array}$ & $\mathrm{CIA}+\mathrm{QoL}$ & $\begin{array}{l}\text { Monocentric } \\
\text { prospective } \\
\text { randomized } \\
\text { open trial }\end{array}$ & $\begin{array}{l}\text { Descriptive } \\
\text { statistics; } \\
\text { Chi-squared } \\
\text { test; per- } \\
\text { protocol } \\
\text { analysis; } \\
\text { Intention-to- } \\
\text { treat (mITT) } \\
\text { sample }\end{array}$ & $\begin{array}{l}\text { N. } 79 \text { (n. } 41 \\
\text { scalp cooling } \\
\text { (CAP) group; } \\
\text { n. } 38 \text { no scalp } \\
\text { cooling } \\
\text { NoCAP } \\
\text { group) }\end{array}$ & $\begin{array}{l}\text { Females >18 } \\
\text { years old; } \\
\text { breast cancer } \\
\text { stage I-III; } \\
\text { ECOG 0-1; } \\
\text { planned } \\
\text { chemotherapy }\end{array}$ & $\begin{array}{l}\text { Dean Scale; } \\
\text { medical staff } \\
\text { rate of } \\
\text { wig/scarf use, } \\
\text { tolerability, } \\
\text { hair pull test, } \\
\text { trichogram; } \\
\text { EORTC QLQ- } \\
\text { C30; BR23 }\end{array}$ & $\begin{array}{l}\text { 1) Hair } \\
\text { preservation } \\
\text { was achieved } \\
\text { in } 39.3 \% \text { of } \\
\text { patients in the } \\
\text { CAP compared } \\
\text { to } 0 \% \text { in the } \\
\text { NoCAP arm in } \\
\text { the per } \\
\text { protocol } \\
\text { analysis (p < } \\
\text { 0.001. } \\
\text { 2) Main } \\
\text { reasons for } \\
\text { drop-out were } \\
\text { hair loss, } \\
\text { adverse } \\
\text { events (CAP), } \\
\text { and } \\
\text { randomization } \\
\text { into control } \\
\text { arm. No } \\
\text { differences in } \\
\text { efficacy } \\
\text { between } \\
\text { anthracycline- } \\
\text { based and } \\
\text { non- } \\
\text { anthracycline- } \\
\text { based } \\
\text { regimens; } \\
3 \text { ) QoL did not } \\
\text { differ between } \\
\text { the study arms }\end{array}$ \\
\hline & $\begin{array}{l}\text { Munzon et } \\
\text { al. (2019) }\end{array}$ & $\begin{array}{l}\text { HAIR LOSS + } \\
\text { QoL }\end{array}$ & $\mathrm{CIA}+\mathrm{QoL}$ & $\begin{array}{l}\text { Prospective } \\
\text { two-stage } \\
\text { design } \\
\text { clinical trial }\end{array}$ & $\begin{array}{l}\text { Descriptive } \\
\text { statistics; } \\
\text { Cohen's } \\
\text { Kappa, }\end{array}$ & $\begin{array}{l}\text { N. } 139 \text { Breast } \\
\text { Cancer } \\
\text { patients }\end{array}$ & $\begin{array}{l}\text { Median age } 47 \\
\text { years (range: } \\
28-65) ; \text { of } \\
\text { female }\end{array}$ & $\begin{array}{l}\text { Primary } \\
\text { endpoint: } \\
\text { Dean's scale. } \\
\text { Secondary }\end{array}$ & $\begin{array}{l}56 \text { out of } 131 \\
\text { evaluated } \\
\text { patients } \\
\text { succesfully }\end{array}$ \\
\hline
\end{tabular}

Page $7 / 17$ 


\begin{tabular}{|c|c|c|c|c|c|c|c|c|c|}
\hline & & & & & $\begin{array}{l}\text { Dean's score; } \\
\text { Repeated } \\
\text { measured } \\
\text { ANOVA; } \\
\text { linear } \\
\text { trasformation } \\
\text { to standarize } \\
\text { raw scores }\end{array}$ & & $\begin{array}{l}\text { patients with } \\
\text { diagnosis of } \\
\text { early-stage } \\
\text { breast cancer } \\
\text { and with a } \\
\text { planned } \\
\text { course of } \\
\text { chemotherapy } \\
\text { in the adjuvant } \\
\text { setting. }\end{array}$ & $\begin{array}{l}\text { endpoints: } \\
\text { Technology } \\
\text { Acceptance } \\
\text { Model (TAM) } \\
\text { Questionnaire, } \\
\text { EORTC-QLQ- } \\
\text { C30, EORTC- } \\
\text { QLQ-BR23, . }\end{array}$ & $\begin{array}{l}\text { prevented HL } \\
\text { ( } 43 \%, 95 \% \text { CI: } \\
34-51 \%) .24 \\
\text { ( } 32 \%) \text { patients } \\
\text { discontinued } \\
\text { the scalp } \\
\text { cooling } \\
\text { because of } \\
\text { alopecia or } \\
\text { scalp-cooling } \\
\text { related AE, } 3 \\
\text { patients had } \\
\text { missing } \\
\text { information on } \\
\text { CIA, and } 48 \\
\text { patients ( } 64 \%) \\
\text { had a HL } \\
\text { greater than } \\
50 \% \text { after CT }\end{array}$ \\
\hline \multirow[t]{4}{*}{$\begin{array}{l}\text { COHORT } \\
\text { STUDY }\end{array}$} & $\begin{array}{l}\text { Rugo et al.. } \\
(2012)\end{array}$ & $\begin{array}{l}\text { HAIR LOSS + } \\
\text { QoL }\end{array}$ & CIA + QoL & $\begin{array}{l}\text { Prospective } \\
\text { study }\end{array}$ & Not specified & $\begin{array}{l}\text { N. } 20 \text { patients } \\
\text { under CTX } \\
\text { regiment }\end{array}$ & $\begin{array}{l}\text { Women with } \\
\text { stage I breast } \\
\text { cancer }\end{array}$ & $\begin{array}{l}\text { Dean Scale; } \\
\text { QoL test; time } \\
\text { to quality of } \\
\text { hair re-growth } \\
\text { and impact of } \\
\text { Hair Loss on } \\
\text { treatment } \\
\text { decisions } \\
\text { questionnaire }\end{array}$ & $\begin{array}{l}19 \text { of } 20 \\
\text { patients (95\%) } \\
\text { completed all } \\
\text { CTX using the } \\
\text { DigniCap } \\
\text { System. By } \\
\text { independent } \\
\text { panel (IP) } \\
\text { assessment, } \\
15 \text { patients } \\
\text { ( } 75 \% \text { ) had a } \\
\text { maximum of } \leq \\
\text { grade } 2 \text { hair } \\
\text { loss }\end{array}$ \\
\hline & $\begin{array}{l}\text { Fehr et al. } \\
\text { (2016) }\end{array}$ & $\begin{array}{l}\text { HAIR LOSS + } \\
\text { SATISFACTION }\end{array}$ & $\begin{array}{l}\text { CIA + } \\
\text { SATISFACTION }\end{array}$ & $\begin{array}{l}\text { Prospective } \\
\text { study }\end{array}$ & $\begin{array}{l}\text { Descriptive } \\
\text { statistics; } \\
\text { multivariate } \\
\text { analyses }\end{array}$ & $\begin{array}{l}\text { N. } 55 \text { female } \\
\text { patients }\end{array}$ & $\begin{array}{l}\text { Cancer } \\
\text { patients=> } 18 \\
\text { years old; } \\
\text { planned } \\
\text { neoadjuvant or } \\
\text { adjuvant } \\
\text { chemotherapy; } \\
\text { breast, } \\
\text { endometrial, } \\
\text { or ovarian } \\
\text { cancer; } \\
\text { patients (N } 42, \\
76 \% \text { breast } \\
\text { cancer; N12, } \\
22 \% \text { ovatian } \\
\text { cancer; N 1, } \\
1.8 \% \\
\text { endometrial } \\
\text { cancer). Mean } \\
\text { age } 56 \text { ds } 12 \\
\text { years (range } \\
32-79 \text { year } \\
\end{array}$ & $\begin{array}{l}\text { Photographs of } \\
\text { the patient's } \\
\text { head; self- } \\
\text { administered } \\
\text { satisfaction } \\
\text { questionnaire; } \\
\text { case report } \\
\text { form using } \\
\text { WHO grading } \\
\text { of acute and } \\
\text { subacute } \\
\text { toxicity for } \\
\text { alopecia }\end{array}$ & $\begin{array}{l}78 \% \\
\text { underwent } \\
\text { scalp cooling } \\
\text { until } \\
\text { completion of } \\
\text { chemotherapy. } \\
\text { Younger } \\
\text { women and } \\
\text { those } \\
\text { receiving } \\
\text { paclitaxel } \\
\text { weekly or } \\
\text { paclitaxel- } \\
\text { carboplatin } \\
\text { experienced } \\
\text { less alopecia }\end{array}$ \\
\hline & $\begin{array}{l}\text { Rugo et al., } \\
\text { (2017) }\end{array}$ & $\begin{array}{l}\text { HAIR LOSS + } \\
\text { QoL }\end{array}$ & CIA + QoL & $\begin{array}{l}\text { Multicenter } \\
\text { prospective }\end{array}$ & $\begin{array}{l}\text { Descriptive } \\
\text { statistics; } \\
\text { Fisher exact } \\
\text { test; exact } \\
\text { method } \\
\text { based on } \\
\text { binomial } \\
\text { distribution; } \\
\text { 2-sided x2 } \\
\text { test; per- } \\
\text { protocol } \\
\text { analysis }\end{array}$ & $\begin{array}{l}\text { N. } 122 \text { (n.106 } \\
\text { in the scalp } \\
\text { cooling } \\
\text { group; n. } 16 \\
\text { in the control } \\
\text { group) }\end{array}$ & $\begin{array}{l}\text { Females }>=18 \\
\text { years old; } \\
\text { stage I or II } \\
\text { breast cancer; } \\
\text { planned } \\
\text { neoadjuvant } \\
\text { cytotoxic } \\
\text { therapy }\end{array}$ & $\begin{array}{l}\text { Dean scale; } \\
\text { Patient } \\
\text { Symptom } \\
\text { Survery; } \\
\text { EORTC-QLQ }\end{array}$ & $\begin{array}{l}\text { Less hair loss } \\
\text { at } 4 \text { weeks } \\
\text { after } \\
\text { treatment. } 3 \text { of } \\
5 \text { quality-of- } \\
\text { life measures } \\
\text { were } \\
\text { significantly } \\
\text { better } 1 \text { month } \\
\text { after the end } \\
\text { of } \\
\text { chemotherapy } \\
\text { in the scalp } \\
\text { cooling group. }\end{array}$ \\
\hline & $\begin{array}{l}\text { Chan et al. } \\
(2018)\end{array}$ & $\begin{array}{l}\text { HAIR LOSS + } \\
\text { MOOD + BODY } \\
\text { IMAGE }\end{array}$ & $\mathrm{CIA}+\mathrm{PSY}$ & $\begin{array}{l}\text { Prospective } \\
\text { study }\end{array}$ & $\begin{array}{l}\text { Descriptive } \\
\text { statistics; } \\
\text { Bonferroni } \\
\text { correction; } \\
\text { paired- }\end{array}$ & $\begin{array}{l}\text { N. } 60 \\
\text { (categorised } \\
\text { according to } \\
\text { chemotherapy } \\
\text { regimen: n. } 20\end{array}$ & $\begin{array}{l}\text { Females >18 } \\
\text { years old; } \\
\text { stage I-III } \\
\text { breast cancer; } \\
\text { recommended }\end{array}$ & $\begin{array}{l}\text { Dean scale; } \\
\text { PHQ-9; GAD-7; } \\
\text { BIS; } \\
\text { information on } \\
\text { the use of any }\end{array}$ & $\begin{array}{l}33 \% \text { of } \\
\text { patients } \\
\text { reported } \\
\text { Minimal Hair } \\
\text { Loss. Patients }\end{array}$ \\
\hline
\end{tabular}

Page 8/17 


\begin{tabular}{|c|c|c|c|c|c|c|c|c|}
\hline & & & & $\begin{array}{l}\text { samples t- } \\
\text { test; logistic } \\
\text { regression; } \\
\text { weighted } \\
\text { kappa score }\end{array}$ & $\begin{array}{l}\text { in Cohort } 1 \\
\text { TC/TCH; n.20 } \\
\text { in Cohort } 2 \\
\text { TAC; n. } 20 \text { in } \\
\text { Cohort } 3 \text { FEC- } \\
\text { D/AC-P) }\end{array}$ & \begin{tabular}{|l} 
to receive \\
adjuvant or \\
neoadjuvant \\
chemotherapy \\
regimens (TC, \\
TCH, TAC, AC, \\
AC-P, FEC-D); \\
conversant in \\
English; \\
provided \\
signed \\
consent; \\
agreed to \\
undergo \\
follow-up for \\
10 years
\end{tabular} & $\begin{array}{l}\text { head covering, } \\
\text { usefulness and } \\
\text { probabily of } \\
\text { cap future use } \\
\text { and adverse } \\
\text { events }\end{array}$ & \begin{tabular}{|l} 
receiving \\
taxane-only \\
chemotherapy \\
had the \\
highest rate of \\
MHL (45\%). \\
Patient- \\
reported \\
anxiety \\
reduced \\
significantly in \\
all patients, \\
but no \\
difference was \\
observed for \\
depression or \\
body image \\
irrespective of \\
degree of hair \\
loss. Scalp \\
cooling relater \\
adverse \\
events were \\
uniformly of \\
low grade and \\
all resolved.
\end{tabular} \\
\hline $\begin{array}{l}\text { Vassalli et } \\
\text { al. (2018) }\end{array}$ & $\begin{array}{l}\text { EFFICACY + } \\
\text { SAFETY + QoL }\end{array}$ & $\mathrm{CIA}+\mathrm{QoL}$ & $\begin{array}{l}\text { Prospective } \\
\text { study }\end{array}$ & $\begin{array}{l}\text { Descriptive } \\
\text { and } \\
\text { inferential } \\
\text { statistics }\end{array}$ & $\begin{array}{l}\text { N. } 49 \text { patients } \\
\text { (mean age } 51 \\
\text { years) }\end{array}$ & \begin{tabular}{|l} 
Women with \\
early breast \\
cancer who \\
receiving \\
neo/adjuvant \\
treatment.
\end{tabular} & \begin{tabular}{|l} 
Dean's \\
alopecia scale; \\
Digital \\
photographs; \\
Visual \\
Analogical \\
Scale (VAS); \\
EORTC QLQ- \\
C30.
\end{tabular} & $\begin{array}{l}\text { 1) Fifteen } \\
\text { patients (30\%) } \\
\text { stopped the } \\
\text { treatment } \\
\text { because of } \\
\text { loss of hair in } \\
9 \text { patients. At } \\
\text { the end of } \\
\text { chemotherapy, } \\
13 \text { patients } \\
\text { (27\%) had no } \\
\text { loss of hair } \\
\text { (Dean score } \\
0 \text { ), 25 patients } \\
\text { (51\%) had a } \\
\text { minimal loss } \\
\text { of hair (Dean } \\
\text { score 1 } \\
\text { 2) There } \\
\text { wasn't a } \\
\text { significant } \\
\text { difference } \\
\text { between mean } \\
\text { score value of } \\
\text { QLQ-C30 at } \\
\text { baseline and } \\
\text { after } \\
\text { chemotherapy } \\
\text { and between } \\
\text { the groups } \\
\text { with and } \\
\text { without hair } \\
\text { loss }\end{array}$ \\
\hline $\begin{array}{l}\text { Giarratano } \\
\text { et al. } \\
(2019)\end{array}$ & $\begin{array}{l}\text { EFFICACY + } \\
\text { TOLERABILITY } \\
\text { + PATIENT } \\
\text { JUDGMENT ON } \\
\text { PERFORMANCE }\end{array}$ & $\begin{array}{l}\text { CIA + PATIENT } \\
\text { JUD } \\
\text { PERFORMANCE }\end{array}$ & $\begin{array}{l}\text { Prospective } \\
\text { study }\end{array}$ & \begin{tabular}{|l} 
Descriptive \\
statistics; $\chi 2$ \\
test; Mann- \\
Whitney test
\end{tabular} & $\begin{array}{l}\text { N. } 135 \\
\text { (categorised } \\
\text { according to } \\
\text { chemotherapy } \\
\text { regimen: n.35 } \\
\text { TC; n.31 Txl; } \\
\text { n. } 43 \text { EC-Txl; } \\
\text { n.26 Txl-EC) }\end{array}$ & \begin{tabular}{|l|} 
Females, \\
range age 27- \\
76, breast \\
cancer stage I- \\
III, ongoing \\
adjuvant/neo- \\
adjuvant CT
\end{tabular} & \begin{tabular}{|l} 
Dean Scale; \\
Patients' self- \\
assessment \\
questionnaries \\
on scalp \\
cooling \\
tolerability and \\
performance
\end{tabular} & $\begin{array}{l}\text { The rate of } \\
\text { success in } \\
\text { preventing } \\
\text { alopecia was } \\
77 \%(104 / 135) \\
\text { at } 3 \text { weeks } \\
\text { from the start } \\
\text { of CT and } 60 \% \\
(81 / 135) \text { at } 3 \\
\text { weeks from } \\
\text { the end of } \\
\text { treatment }\end{array}$ \\
\hline
\end{tabular}

Page 9/17 


\begin{tabular}{|c|c|c|c|c|c|c|c|c|c|}
\hline & & $\begin{array}{l}\text { ANXIETY } \\
\text { DEPRESSION + } \\
\text { SAFETY }\end{array}$ & & & $\begin{array}{l}\text { rank sum } \\
\text { test. }\end{array}$ & & $\begin{array}{l}\text { Cancer; } \\
\text { planned adj CT }\end{array}$ & $\begin{array}{l}\text { Common } \\
\text { Terminology } \\
\text { Criteria for } \\
\text { Adverse Events } \\
\text { version } 4.0 \\
\text { (CTCAE v 4.0). } \\
\text { Comfort } \\
\text { analogic scale } \\
\text { (AS) for } \\
\text { tolerability. An } \\
\text { AS for pain } \\
\text { evaluation. } \\
\text { Hospital } \\
\text { Anxiety and } \\
\text { Depression } \\
\text { Scale (HADS) }\end{array}$ & $\begin{array}{l}\text { pts }(11 \%) \\
\text { prematurely } \\
\text { interrupted } \\
\text { use of the } \\
\text { scalp-cooling } \\
\text { device due to } \\
\text { inefficacy. } \\
\text { After the last } \\
\text { cycle of } \\
\text { chemotherapy, } \\
\text { the number of } \\
\text { patient who } \\
\text { perceived a } \\
\text { HL < grade } 2 \\
\text { was } 16 \text { (59\%). } \\
\text { The hair } \\
\text { retention } \\
\text { reported by } \\
\text { operators has } \\
\text { been higher } \\
\text { ( } 78 \% \text { ). } 81.5 \% \\
\text { of patients } \\
\text { well tolerated } \\
\text { the treatment. }\end{array}$ \\
\hline & $\begin{array}{l}\text { Orlando et } \\
\text { al., (2019) }\end{array}$ & $\begin{array}{l}\text { HAIR LOSS + } \\
\text { QoL }\end{array}$ & CIA + QoL & $\begin{array}{l}\text { Prospective } \\
\text { study }\end{array}$ & $\begin{array}{l}\text { Descriptive } \\
\text { statistics }\end{array}$ & $\begin{array}{l}\text { n.127 Breast } \\
\text { Cancer } \\
\text { patients }\end{array}$ & $\begin{array}{l}\text { Early-stage BC } \\
\text { candidacy to } \\
\text { neo/adjuvant } \\
\text { anthracycline } \\
\text { and/or taxane } \\
\text { chemotherapy; } \\
\text { age range 31- } \\
75 \text { yo }\end{array}$ & $\begin{array}{l}\text { Dean's } \\
\text { alopecia scale, } \\
\text { EORTC QLQ- } \\
\text { C30 and BR-23 }\end{array}$ & $\begin{array}{l}\text { The success } \\
\text { rate in } \\
\text { preventing CIA } \\
\text { was } 71.7 \% \text {. } \\
\text { The use of } \\
\text { head cover } \\
\text { (wig, cap or } \\
\text { scarf) was } \\
\text { very low } \\
\text { (29.9\%). The } \\
\text { tolerability } \\
\text { was excellent, } \\
\text { with low rate } \\
\text { of withdrawal } \\
\text { (17.3\%), } \\
\text { mainly due to } \\
\text { unsatisfactory } \\
\text { hair } \\
\text { preservation } \\
\text { (9.4\%). } \\
\end{array}$ \\
\hline $\begin{array}{c}\text { Basic } \\
\text { Research }\end{array}$ & $\begin{array}{l}\text { Campos- } \\
\text { Gomez, et } \\
\text { al. (2016) }\end{array}$ & $\begin{array}{l}\text { EFFICACY + } \\
\text { SAFETY + QoL }\end{array}$ & CIA + QoL & $\begin{array}{l}\text { Observational } \\
\text { study }\end{array}$ & $\begin{array}{l}\text { Descriptive } \\
\text { and } \\
\text { inferential } \\
\text { statistics }\end{array}$ & $\begin{array}{l}\text { N. } 210 \text { women } \\
\text { with breast } \\
\text { cancer (n. } 110 \\
\text { in scalp- } \\
\text { cooling } \\
\text { system group; } \\
\text { n. } 100 \text { in no } \\
\text { scalp-cooling } \\
\text { group) }\end{array}$ & $\begin{array}{l}\text { Women with } \\
\text { breast cancer } \\
\text { receiving neo } \\
\text { or adjuvant }\end{array}$ & $\begin{array}{l}\text { World Health } \\
\text { Organization } \\
\text { (WHO) } \\
\text { criteria; Photo } \\
\text { documentation; } \\
\text { EORTC QLQ- } \\
\text { C30; EORTC- } \\
\text { QLQ-BR23 }\end{array}$ & $\begin{array}{l}\text { 1) Nurses' } \\
\text { ratings } \\
\text { indicated that } \\
\text { hair loss } \\
\text { frequency was } \\
\text { constantly } \\
\text { lower, at each } \\
\text { cycle of } \\
\text { chemotherapy. } \\
\text { 2) Differences } \\
\text { between the } \\
\text { two groups } \\
\text { were } \\
\text { statistically } \\
\text { significant at } \\
\text { cycles } 1 \text { and } 4 \\
\text { ( P < 0.047). 3) } \\
\text { higher well- } \\
\text { being was } \\
\text { found in } \\
\text { successfully } \\
\text { scalp-cooled } \\
\text { patients, as } \\
\text { indicated by a } \\
\text { general better } \\
\text { health-related } \\
\text { quality of life, } \\
\text { whereas } \\
\text { unsuccessfully } \\
\text { scalp-cooled }\end{array}$ \\
\hline
\end{tabular}




\begin{tabular}{|c|c|c|c|c|c|c|c|c|}
\hline & & & & & & & & \begin{tabular}{|l} 
patients \\
reported \\
lowest well- \\
being
\end{tabular} \\
\hline $\begin{array}{l}\text { Friedrichs } \\
\text { et al. } \\
(2014)\end{array}$ & $\begin{array}{l}\text { HAIR LOSS + } \\
\text { SATISFACTION } \\
+ \text { ADVERS } \\
\text { EFFECTS }\end{array}$ & $\begin{array}{l}\text { CIA + } \\
\text { SATISFACTION } \\
+ \text { SAFETY }\end{array}$ & $\begin{array}{l}\text { Observational } \\
\text { study }\end{array}$ & $\begin{array}{l}\text { Descriptive } \\
\text { statistics }\end{array}$ & N. 83 patients & \begin{tabular}{|l|} 
Females \\
$>=18$ years \\
old with breast \\
cancer \\
diagnosis
\end{tabular} & \begin{tabular}{|l} 
VAS; patient's \\
information on \\
head cover; \\
hair loss and \\
regrowth rating \\
by an expert \\
based on a set \\
of \\
photographs; a \\
modified \\
version of \\
Dean's scale
\end{tabular} & \begin{tabular}{|l}
$64 / 83$ (77\%) \\
patients \\
finished CT \\
and SC \\
treatment. 19 \\
patients \\
dropped-out \\
due to \\
unspecified \\
intolerance \\
(10.8\%), hair \\
loss (6.0\%), \\
cancer related \\
emergency \\
cases or \\
disease \\
progression \\
(3.6\%) and SC- \\
related side \\
effects (2.4\%) \\
(feeling cold, \\
headache). \\
\end{tabular} \\
\hline $\begin{array}{l}\text { Peerbooms, } \\
\text { etal., } \\
(2015)\end{array}$ & $\begin{array}{l}\text { ATTITUDE AND } \\
\text { KNOWLEDGE } \\
\text { ON SC }\end{array}$ & $\begin{array}{l}\text { ATTITUDE AND } \\
\text { KNOWLEDGE } \\
\text { ON SC }\end{array}$ & $\begin{array}{l}\text { Observational } \\
\text { study }\end{array}$ & $\begin{array}{l}\text { Descriptive } \\
\text { statistics }\end{array}$ & $\begin{array}{l}\text { N.177 } \\
\text { patiens, N.49 } \\
\text { nurses, N.100 } \\
\text { MDs }\end{array}$ & $\begin{array}{l}\text { Ex breast } \\
\text { cancer scalp- } \\
\text { cooled and } \\
\text { nonscalp- } \\
\text { cooled } \\
\text { patients, } \\
\text { nurses and } \\
\text { Medical } \\
\text { Doctors from } \\
\text { ScoolingCap } \\
\text { and non-SC } \\
\text { hospitals }\end{array}$ & $\begin{array}{l}\text { self-developed } \\
\text { questionnaires }\end{array}$ & $\begin{array}{l}\text { The majority of } \\
\text { MDs and } \\
\text { nurses were } \\
\text { satisfied with } \\
\text { the results of } \\
\text { SC, as were } \\
\text { SC patients. } \\
\text { Seventy } \\
\text { percent of SC } \\
\text { patients with } \\
\text { insufficient } \\
\text { results } \\
\text { (20/52) } \\
\text { reported to } \\
\text { mind it very } \\
\text { much. With } \\
\text { expected } \\
\text { success rates } \\
\text { of } 35 \% \text { and } \\
50 \%,\end{array}$ \\
\hline
\end{tabular}




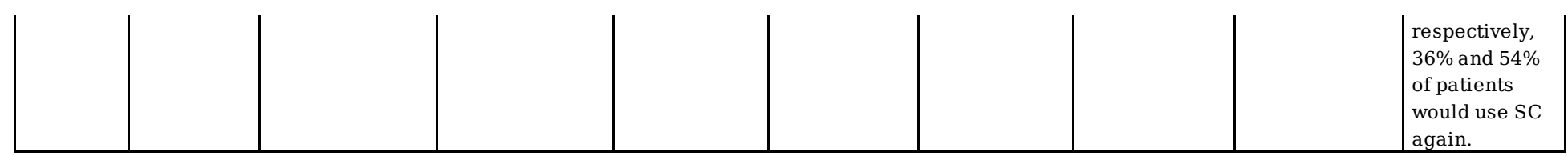

In cohort study designs (Rugo et al., 2012; Fehr et al., 2016; Rugo et al., 2017; Vassalli et al., 2018; Chan et al., 2019; Giarratano et al., 2019; Prochilo et al., 2019; Orlando et al., 2019) prospective observational studies have been conducted mostly in multicentric research, focusing on CIA and quality of life, satisfaction, and patient-reported performance variables in an oncological population diagnosed with breast cancer. All studies reported a good rate of success for the DigniCap technology associated with chemotherapy treatment, particularly, regarding hair loss (average: $25 \%$ of CIA). The included studies reported an average of $30 \%$ drop-out rate from the scalp cooling application. Finally, an average of $10 \%$ of patients reported adverse events (headache) and/or were discontinued from the treatment due to feeling cold. Regarding the positive psychological impact of the DigniCap technology, preventing CIA and improving the quality of life was not significantly detected. Vassalli et al. (2018) reported no significant changes in the quality of life pre- and post-treatment, and even reported an increase in fatigue; by contrast, only anxiety seemed to have decreased in this cohort. Similarly, Pochilo et al. (2019) reported a decrease in anxiety level with an increase in depressive thoughts.

The basic research category (Friedrichs et al., 2014; Campos-Gomez et al., 2015; Peerbooms et al., 2015; Shaw et al., 2016) are composed of three observational studies and one qualitative study. Observational studies detected higher well-being in successfully scalp-cooled patients, as indicated by a generally better health-related quality of life associated with the conservative effect of the DigniCap technology for hair loss. A qualitative study offered a thematic analysis of the patient experience of scalp cooling: the promise of faster regrowth seemed a motivator for adherence to the DigniCap application.

In Table 3, review studies ( $n=2)$ are reported. Breed's review (2011) focused on the physical impact of DigniCap technology in chemotherapy, analysing hair loss measurements. Further, the review offered a general overview of CIA and scalp cooling. Subsequently, Marks et al. (2019) conducted a systematic review to evaluate the use of scalp cooling for CIA and quantitative quality of life measures. The comprehensive analysis evidenced by a few studies (4 of the 13 studies published before October 2018) concluded that scalp cooling was associated with significant improvements in quality of life measures; 8 (62\%) determined that there were either non-significant or no improvements, and 1 (7.7\%) provided a mixed conclusion. Although 2 (50\%) randomised controlled trials demonstrated that scalp cooling can effectively prevent CIA depending on the chemotherapy regimen, these studies did not show that successful hair preservation was associated with improved quality of life measures.

Table 3 - Details of review paper 


\begin{tabular}{|c|c|c|c|c|c|c|c|c|}
\hline $\begin{array}{c}\text { TYPE OF } \\
\text { STUDY }\end{array}$ & PAPER & $\begin{array}{c}\text { KEYWORD } \\
\text { AIM }\end{array}$ & TOPIC & $\begin{array}{c}\text { STUDY } \\
\text { DESIGN }\end{array}$ & SAMPLE SIZE & $\begin{array}{l}\text { TARGET } \\
\text { SAMPIE }\end{array}$ & TESTS & OUTCOMES \\
\hline \multirow[t]{2}{*}{$\begin{array}{c}\text { REVIEW } \\
\text { STUDIES } \\
\text { (N. 2) }\end{array}$} & $\begin{array}{l}\text { Marks } \\
\text { et al. } \\
\text { (2019). }\end{array}$ & $\begin{array}{l}\text { QoL + } \\
\text { ANXIETY } \\
\text { DEPRESSION } \\
+ \text { BODY } \\
\text { IMAGE }\end{array}$ & $\begin{array}{l}\text { QoL } \\
+ \text { PSY }\end{array}$ & $\begin{array}{l}\text { Systematic } \\
\text { review }\end{array}$ & $\begin{array}{l}13 \text { studies ( } 4 \text { randomized } \\
\text { clinical trials, } 8 \text { cohort } \\
\text { studies, and } \\
1 \text { cross-sectional study) }\end{array}$ & $\begin{array}{l}\text { Primary clinical } \\
\text { studies that } \\
\text { reported on a } \\
\text { quantitative } \\
\text { QoL measure. } \\
\text { All studies } \\
\text { investigated } \\
\text { use of scalp } \\
\text { cooling to } \\
\text { prevent CIA in } \\
\text { breast cancer } \\
\text { patients, and } \\
\text { the majority } \\
\text { included a } \\
\text { control group of } \\
\text { non scalp- } \\
\text { cooled patients. }\end{array}$ & $\begin{array}{l}\text { EORTC QLQ- } \\
\text { C30, QLQ- } \\
\text { BR23, GAD, } \\
\text { Study- } \\
\text { specific } \\
\text { questionnaire } \\
\text { on distress, } \\
\text { HADS, BIS, } \\
\text { MBA, PHQ-9, } \\
\text { VAS, WHO-5. }\end{array}$ & $\begin{array}{l}4 \text { ( } 31 \% \text { ) of the } 13 \text { studies concluded } \\
\text { that scalp cooling was associated } \\
\text { with significant improvements in } \\
\text { QoL measures; } 8 \text { ( } 62 \% \text { ) determined } \\
\text { that there was either non-significant } \\
\text { or no improvements; and } 1 \text { ( } 7.7 \%) \\
\text { provided a mixed } \\
\text { conclusion. Although } 2 \text { (50\%) RCT } \\
\text { demonstrated that scalp cooling can } \\
\text { effectively prevent CIA depending on } \\
\text { the chemotherapy regimen, these } \\
\text { studies did not show that successful } \\
\text { hair preservation was associated with } \\
\text { improved QoL measures. }\end{array}$ \\
\hline & $\begin{array}{l}\text { Breed } \\
\text { et al. } \\
(2011 .\end{array}$ & HAIR LOSS & CIA & $\begin{array}{l}\text { Expert } \\
\text { Review }\end{array}$ & $\begin{array}{l}\text { N.58 scalp cooling } \\
\text { publications and n.3 } \\
\text { personal communications } \\
\text { (n.7 randomized studies, } \\
\text { with and without scalp } \\
\text { cooling; n.6 } \\
\text { nonrandomized studies } \\
\text { with statistical analysis; } 11 \\
\text { nonrandomized studies } \\
\text { with control groups, } \\
\text { without statistical } \\
\text { analysis; n.36 studies } \\
\text { without controls) }\end{array}$ & Not specified & $\begin{array}{l}\text { patient's } \\
\text { information } \\
\text { on head } \\
\text { cover; hair } \\
\text { loss and } \\
\text { regrowth } \\
\text { rating by an } \\
\text { expert based } \\
\text { on a set of } \\
\text { photographs; } \\
\text { a modified } \\
\text { version of } \\
\text { Dean's scale }\end{array}$ & $\begin{array}{l}\text { Scalp cooling is by far the best } \\
\text { method to reduce CIA. Concerns } \\
\text { about the protection of malignant } \\
\text { cells in the scalp skin by scalp } \\
\text { cooling have been proven to be } \\
\text { exaggerated. The majority of patients } \\
\text { tolerate scalp cooling very well. } \\
\text { Scalp cooling is cost-effective when } \\
\text { compared with purchasing wigs and } \\
\text { other head covers. Scalp cooling is } \\
\text { effective but not for all chemotherapy } \\
\text { patients. }\end{array}$ \\
\hline
\end{tabular}

The screened reviews showed increasing interest in the DigniCap technology over time, and implemented investigations to analyse the magnitude of impact on mental health of oncological patients during and after chemotherapy intervention.

\section{Discussion And Conclusion}

The aim of this scoping review was to map and determine the current literature regarding the mental health impact of DigniCap technology applied to chemotherapy treatment in terms of quality of life and wellness of oncological patients. Considering the exclusion/inclusion criteria, a total of 17 articles were included in the scoping review process.

The DigniCap technological solution was designed to reduce the distressing factor induced by CIA as a side-effect of exposure to chemotherapy in oncological patients. This innovative solution aimed to be a preventive intervention that could improve patient care.

This review has demonstrated the lack of research conducted on the efficacy of the DigniCap system adoption as preventive care for the well-being of patients through the prevention of their hair loss. Progressively, researchers have investigated the efficacy of the DigniCap technology for measuring physical preservation, taking into account the rate of hair loss and measuring the degree of satisfaction. Innovative solutions should be better studied by considering mental health-related effects during pharmacological treatment and post-treatment. Our results showed a lack of investigation focused on well-being and emotional impact indexes, as well as the patient-oriented perspective for quality of life. The implementation of research in this area, particularly, the progress in the number of research protocols on this topic, are increasing. Future research should address these key points:

1. DigniCap-targeted aims for mental efficacy

2. Consent about what constitutes a successful rate during DigniCap use

3. Mental health outcomes of DigniCap use 
DigniCap-targeted aims for mental efficacy. The main objective of this study was to evaluate hair quantity (preserved or lost) as a measure of quality of life in terms of mental health and well-being. According to Marks et al. (2019), the correlation between successful hair preservation and improved quality of life has not yet been verified, even if studies focused on this topic apply different evaluation systems. Targeted aims should be addressed and tailored to develop DigniCap-specific measurements for the quality of life of cancer patients. Moreover, the associated effect of DigniCap application should be expanded to the evaluation of the patient's mental health, and not just hair measurements. Over time follow-ups could be able to explain mental health efficacy and/or adverse effects.

Consent about what constitutes a successful rate during DigniCap use. The scoping review highlighted the lack of consent regarding what constitutes the scalp cooling success rate. Researchers have applied evaluation processes based on various tests and measures: hair preservation rate, fast regrowth rate, growth rate time, heterogeneous patient reports, and medical reports. However, future research should focus on mental health effects, paying attention to the personality, psychological traits, and social determinants of patients as influencing factors for the successful impact of the technological solution (DigniCap) in chemotherapy. Qualitative and quantitative assessments would address compound wellness and quality of life endpoints.

Mental health outcomes of DigniCap use. The efficacy of the DigniCap system should be better tested. Preventive interventions for better adherence and successful effects of DigniCap treatment could be performed. Structured and accurate informative psychological mediation could be developed to mitigate the negative effects (mood issues) and boost the potential positive mental impact on quality of life and well-being during treatment, and even more after the treatment.

Clinical Implication. Guidelines and recommendations might be identified in order to reduce unexpected psychological distress, personal unmodulated expectations, drop-outs, and a lower sense of well-being. Lastly, aesthetic learning and self-caring should be tested as an adjuvant individual action to improve the psychological adaptation process of patients to the intensive clinical treatment, preventing mental distress during medical pathways.

Limitations. The limitation of this review is the low number of studies that met our inclusion criteria. This limit highlights the need to improve research interest in this topic, as well as on the direct and quick impact to the clinical practice of oncological treatments. A better tailored clinical pathway can enhance the exploitation of the potential of innovative technological solutions in chemotherapy.

\section{Declarations}

\section{Funding}

This study did not receive funding from any source.

\section{Conflict of Interest}

The authors declare no conflicts of interest.

Availability of data and material: Data will be available behind request to the Authors

\section{Code availability: N/A}

Authors' contributions: RJ elaborated data, GF statistical analysis, DDG conceptualization

Ethics approval: N/A

Consent to participate: N/A

Consent for publication: N/A

\section{References}


Arksey H, O'Malley L. (2005) Scoping studies: towards a methodological framework. International Journal of Social Research Methodology8(1),19-32.

Breed, W.P.M., Van den Hurk, C.J.G., \& Peerbooms M. (2011). Presentation, impact and prevention hair loss: scalp cooling potentials and limitations. Expert Review Dermatolology, 6, 109-125. https://doi.org/10.1586/edm.10.76

Campos-Gomez S, Claudis-Morales N, Campos-Gómez KA, Olivares-Mendoza B, García-Garcés M, Rojas-Cruz L. (2016) Chemotherapy-induced alopecia prevention and effects on quality of life among women with breast cancer: A study of a scalpcooling system used in a Mexican public hospital. [abstract]. In: Proceedings of the Thirty-Eighth Annual CTRC-AACR San Antonio Breast Cancer Symposium: 2015 Dec-8-12; San Antonio, TX. Philadelphia (PA): AACR; Cancer Res 76; P1-11-05

Chan, A., Bauwens, A., Pontre, S., Jackson, S., McGlone, F., Ernenwein, T., Chih, J., Reid, C. (2018). Efficacy of scalp cooling in reducing alopecia in early breast cancer patients receiving contemporary chemotherapy regimens The Breast41, 127-132 doi: 10.1016/j.breast.2018.07.006

Cigler T, Melin SA, Klein P, Hurvitz SA, Melisko M, Moore A, Park GD, Bageman E, Ver Hoeve ES, Rugo HS. (2017) Body image in women with breast cancer using a scalp cooling system to reduce chemotherapy induced alopecia [abstract]. In: Proceedings of the 2016 San Antonio Breast Cancer Symposium 6-10; San Antonio, TX. Philadelphia (PA): AACR; Cancer Res 2017, 77

Di Giacomo, D., Ranieri, J., Perilli, E., ...Passafiume, D., Ficorella, C. (2019) Psychological impact of clinical treatment after breast cancer diagnosis in younger patients (38-50 age range): An explorative 3-year observational study. Neurology Psychiatry and Brain Research 32, 85-90

Fehr, M.K., Welter, J., Sell, W., Jung, R., \& Felberbaum, R. (2016). Sensor-controlled scalp alopecia to prevent chemotherapyinduced alopecia in female cancer patients. Current Oncology 23, e576-e582 https://doi.org/10.3747/co.23.3200

Friedrichs, K., Cartesen, M.H. (2014). Successful reduction of alopecia induced by anthracycline and texane containing adjuvant chemotherapy in breast cancer - clinical evaluation of sensor-controlled scalp cooling. Springerplus3, 500. doi: 10.1186/21931801-3-500

Giarratano, T., Frezzini, S., Zanocco, M., Giorgi, C. A., Mioranza, E., Miglietta, F., Griguolo, G., Falci, C., Faggioni, G., Tasca, G., Di Liso, E., Vernaci, G., Menichetti, A., Mantiero, M., Grosso, D., Guarneri, V., Dieci, M. V. (2019). Use of scalp cooling device to prevent alopecia for early breast cancer patients receiving chemotherapy: A prospective study. Breast Journa/100, 1-6. doi:

$10.1111 /$ tbj. 13711

Linden W, Vodermaier A, Mackenzie R, Greig D. Anxiety and depression after cancer diagnosis: prevalence rates by cancer type, gender, and age. J Affect Disord (2012) 141(2-3):343-51. doi: 10.1016/j.jad.2012.03.025

Marks, D.H., Okhovat, J.P., Hagigeorges, D., et al. (2019). The effect of scalp cooling on CIA-related quality of life in breast cancer patients: a systematic review. Breast Cancer Research Treatment175(2), 267-276. doi:10.1007/s10549-019-05169-0

Mehnert A, Hartung TJ, Friedrich M, et al. (2018) One in two cancer patients is significantly distressed: Prevalence and indicators of distress. Psychooncology27(1):75-82. doi:10.1002/pon.4464

Orlando L., Loparco D., Fedele P., Schiavone P, Quaranta A, Caliolo C, Cinefra M, Rizzo P, Calvani N, Morleo A, Varriano R, BonusoV, Falcone LL, Caloro M, Cinieri S. (2019) Final results of prospective study of scal cooling in preventing chemotherapy-induced alopecia. Future Oncology3(epub) DOI: 10.2217/fon-2019-0066

Peerbooms, M., van den Hurk C.J.G., Breed, W.P.M. (2015). Familiarity, opinions, experience and knowledge about scalp cooling: a Dutch survey among breast cancer patients and oncological professionals. Asian Pacific Journal of Oncology Nursing 2(1), 3541. DOI:

$10.4103 / 2347-5625.152404$ 
Prochilo T., Huscher A., Andreis F., Mirandola M., Zaina E., Pomentale B., Pedrali C., Zanotti L., Mutti S., Zaniboni A. (2019) Hair loss Prevention by a Scalp Cooling Device in Early Breast Cancer Patients:The Poliambulanza Preliminary Experience. Reviews on Recent Clinical Trials14:1-6. doi: 10.2174/1574887113666181120111104

Rugo, H., Serrurier, K. M., Melisko, M., Glencer, A., Hwang, J., D'Agostino, R., Hutchens, S., Esserman, L. J., Melin, S. (2012). Use of the DigniCap System to Prevent Hair Loss in Women Receiving Chemotherapy (CTX) for Stage I Breast Cancer (BC). Cancer Research, 72(24 Supplement) doi: 10.1158/0008-5472.SABCS12-P2-12-11

Rugo, H.S., Klein, P., Melin, S.A., Hurvitz, S.A, Melisko, M.E., Moore, A., Park, G., Mitchel, J., Bageman, E., D'Agostino, R. b., Jr, Ver Hoeve, E.S., Esserman, L., \& Cigler, T. (2017). Association Between Use of a Scalp Cooling Device and Alopecia After Chemotherapy for Breast Cancer. JAMA 317(6), 606-614. doi:10.1001/jama.2016.21038

Seiler, A., \& Jenewein, J. (2019). Resilience in Cancer Patients. Frontiers in psychiatry, 10, 208.

https://doi.org/10.3389/fpsyt.2019.00208

Shaw, J., Baylock, B., O'Reilly, A., Winstanley, J., Pugliano, L., Andrews, K., Boyle, F. (2016). Scalp cooling: a qualitative study to asses the perceptions and experiences of Australian patients with breast cancer. Supportive Care in Cancer24(9), 3813-3820. doi: $10.1007 / \mathrm{s} 00520-016-3206-2$

Tricco AC, Lillie E, Zarin W, O'Brien KK, Colquhoun H, Levac D, et al. (2018) PRISMA Extension for Scoping Reviews (PRISMA-ScR): Checklist and Explanation. Annual International Medicine169(7), 467-73. https://doi.org/10.7326/M18-0850 PMID: 30178033

Vassalli, L., Pedersini, R., Claps, M., Fornaro, C., Conti, E., Tagliani, M., Baronchelli, A., Ragni, D., Lombardi, E., Rodella, F., Amoroso, V., Berruti, A., Simoncini, E. L. (2018). Efficacy and patient acceptability of the DigniCap ScalpCooler to prevent hair loss in breast cancer patients receiving adjuvant chemotherapy. Cancer Research, 78(4 Supplement), P6-11-19. doi:10.1158/1538-

7445.SABCS17-P6-11-19.

\section{Figures}




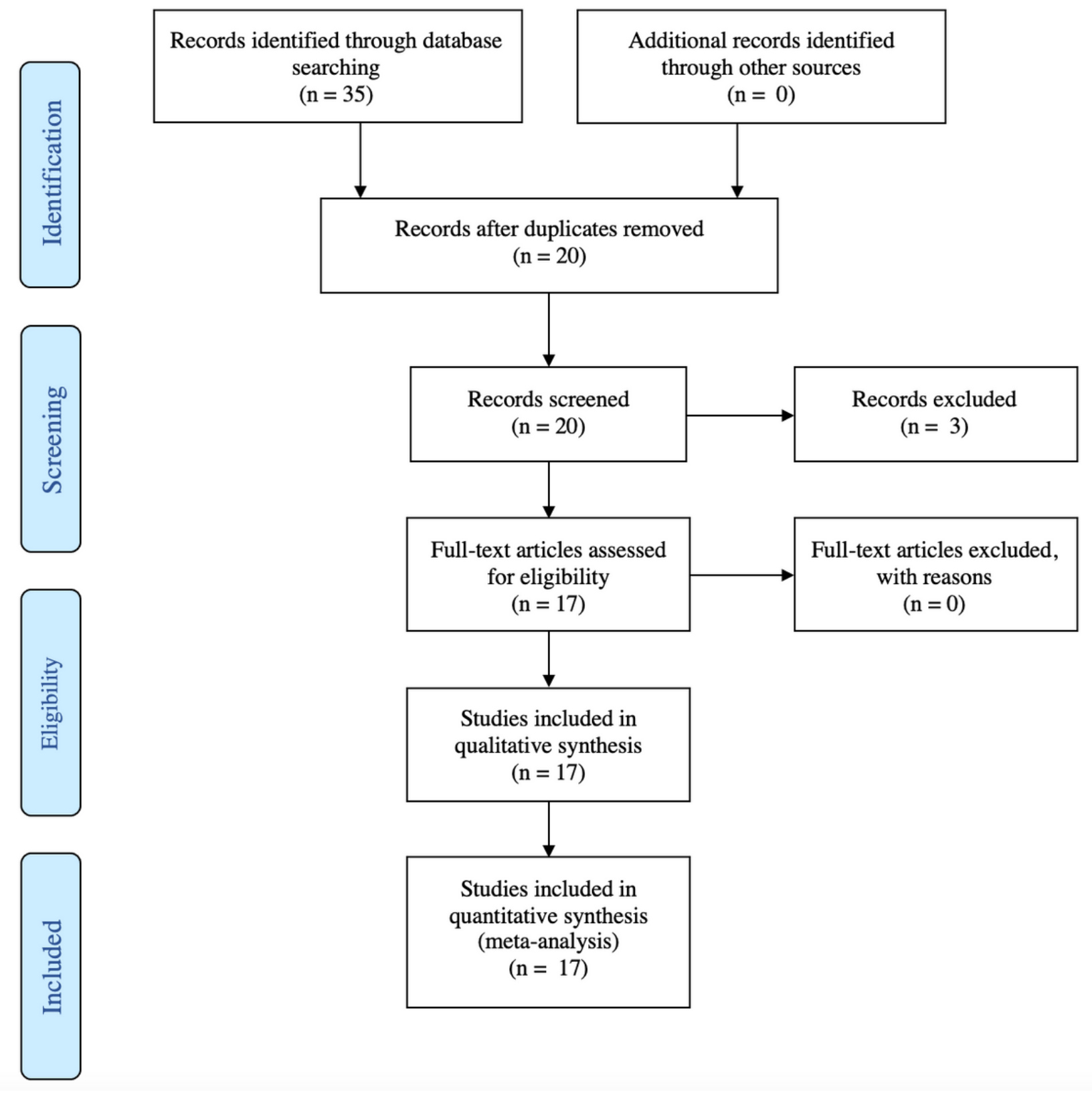

Figure 1

PRISMA Flowchart of selection process for the scoping review of DigniCap system articles/studies until May 2020 